\title{
Aberrant SLC6AI4 Expression Promotes Proliferation and Metastasis of Colorectal Cancer via Enhancing the JAK2/STAT3 Pathway
}

This article was published in the following Dove Press journal: OncoTargets and Therapy

\author{
Hongli Mao' \\ Jinxiu Sheng' \\ Jinlin Jia' \\ Chang Wang' \\ Shanfeng Zhang ${ }^{2}$ \\ Hongle $\mathrm{Li}^{3}$ \\ Fucheng $\mathrm{He} \mathbb{D}^{\prime}$ \\ 'Department of Medical Laboratory, The \\ First Affiliated Hospital of Zhengzhou \\ University, Zhengzhou, Henan, People's \\ Republic of China; ${ }^{2}$ The Laboratory Center \\ for Basic Medicine, School of Basic Medical \\ Sciences, Zhengzhou University, Zhengzhou, \\ Henan, People's Republic of China; \\ ${ }^{3}$ Department of Molecular Pathology, The \\ Affiliated Cancer Hospital of Zhengzhou \\ University, Zhengzhou, Henan, People's \\ Republic of China
}

Background: Solute carrier family 6 member 14 (SLC6A14) is a high-capacity amino acid transporter in mammalian cells. It has gained increasing attention for its potential involvement in the progression and metabolic reprogramming of various malignant tumors. However, the role of SLC6A14 in colorectal cancer (CRC) remains unclear.

Methods: Real-time polymerase chain reaction (qRT-PCR), immunoblotting and immunohistochemistry were carried out to detect the expression level of SLC6A14 in human CRC tissues and CRC-derived cell lines. HCT-116 and Caco-2 cell lines were selected to conduct in vitro functional studies. Cell Counting Kit-8 (CCK-8), colony formation, flow cytometry, cell migration and invasion assays were performed to investigate the role of SLC6A14 in CRC cells. Besides, azoxymethane/dextran sulfate sodium salt (AOM/DSS)-induced CRC and tumor xenograft models were constructed to explore the effects of SLC6A14 blockade or overexpression during tumor progression in vivo.

Results: SLC6A14 was substantially increased in human CRC samples and higher levels of SLC6A14 was correlated with advanced tumor stage, lymph node metastasis and dismal survival of CRC patients. SLC6A14 markedly promoted cell growth, inhibited cell apoptosis, and exacerbated migration and invasion of CRC cells in vitro. Mechanistically, SLC6A14 aggravated these malignant phenotypes through activating JAK2/STAT3 signaling pathway, and inhibiting JAK2/STAT3 signaling with specific inhibitors could reverse SLC6A14-mediated tumorigenic effects. Besides, two different animal studies verified the tumor-promoting effect of SLC6A14 in CRC.

Conclusion: Our data illustrated the crucial function of SLC6A14 during CRC progression, suggesting SLC6A14/JAK2/STAT3 axis may serve as novel therapeutic targets for patients with CRC.

Keywords: SLC6A14, colorectal cancer, CRC, proliferation, migration, STAT3

\section{Introduction}

As one of the most common malignancies in human digestive system, colorectal cancer (CRC) represents a significant contributor to cancer-related deaths and remains a serious health problem worldwide. ${ }^{1}$ However, the pathogenesis of CRC has not been fully elucidated. Increasing evidence suggested that the etiology of CRC is related to multifactorial and complex risk factors including genetic disorders, diet, lifestyles and immunological disorders, apart from age and gender. ${ }^{2,3}$ To date, targeting a series of aberrant oncogenes and their related regulatory pathways could be an optimal option for developing therapeutics and improving the survival rate of CRC patients.
Correspondence: Fucheng He

Department of Medical Laboratory, The First Affiliated Hospital of Zhengzhou University, Zhengzhou 450052, Henan,

People's Republic of China

Email hefucheng@zzu.edu.cn 
Mounting evidence has demonstrated the broad biological functions that amino acids played in tumor growth and metabolism, making amino acid transportation and utilization become the forefront of cancer-cell metabolism in recent years. ${ }^{4}$ Due to the hydrophilic nature, amino acids must rely on specific transporters to enter into the plasma membrane and intracellular membranes to participate in cell metabolism of mammalian cells. Currently, all amino acid transporters in mammalian cells belong to the solute carrier (SLC) gene family. ${ }^{5}$ To satisfy the incremental amino acid demands for tumor metabolism, different tumor cells selectively upregulated different transporters. Previously studies have identified four dramatically increased amino acid transporters in various cancers, including SLC1A5, SLC6A14, SLC7A5 and SLC7A11. ${ }^{6-10}$ SLC6A14 particularly has the most widespread substrate selectivity (including all neutral amino acids and two cationic amino acids) in mammalian cells, thus serving as a high-capacity type of protein which transports all essential amino acids. SLC6A14 has gained increasing attention for its upregulation in a series of malignant tumors including pancreatic cancer, ${ }^{11}$ cervical cancer, ${ }^{12}$ estrogen receptor (ER)-positive breast cancer ${ }^{9}$ and colon cancer, ${ }^{13}$ attested to its importance in tumor biology. Babu et al have shown that crossing the spontaneous breast cancer mice with Slc6a14 ${ }^{-/}$mice significantly inhibited the incidence and progression of breast cancer. ${ }^{14}$ However, the potential role and underlying molecular mechanisms of SLC6A14 in CRC development are still unclear.

In this study, we explored the biological function, clinical significance and molecular mechanisms of SLC6A14 in CRC progression. We found the aberrantly upregulated SLC6A14 markedly accelerated cell proliferation and colony-formation, suppressed cell apoptosis, promoted migration and invasion abilities of CRC cells in vitro. Most of the activated signaling pathways are driven by certain oncogenes, which transform normal cells towards malignant phenotypes, such as uncontrolled proliferation and escape from apoptosis. We carried out Gene Set Enrichment Analysis (GSEA) using TCGA datasets of CRC patients and found that the Janus kinase 2 (JAK2)/signal transducer and activator of transcription 3 (STAT3) signaling was one of the most prominently altered pathways in CRC patients with increased SLC6A14 expression. Moreover, accumulating researches have shown that the abnormalities of JAK2/STAT3 signaling are closely associated with the oncogenesis of CRC. ${ }^{15}$
Modulating JAK2/STAT3 pathway significantly inhibited the growth, induced apoptosis and enhanced antitumor immunities in several experimental mice tumor models. ${ }^{16}$ Additionally, owing to the activated STAT3 signaling cascade finally leads to numerous target genes expression involving tumor cell proliferation, angiogenesis, metastasis and immune escape. STAT3 is a promising drug target for antitumor immunotherapy. ${ }^{17}$ Herein, we found SLC6A14 facilitated the malignant phenotypes of CRC cells by enhancing JAK2/STAT3 signaling pathway. Suppressing JAK2/STAT3 pathway with specific inhibitors BP-1-102 and FLLL32 partly reversed SLC6A14mediated malignant phenotypes in CRC. Collectively, our data identified a pivotal oncogenic role of SLC6A14 in $\mathrm{CRC}$ and highlighted the functional significance of the SLC6A14/JAK2/STAT3 axis in CRC cells.

\section{Patients and Methods Patients and Specimens}

Human colorectal mucosal biopsies were obtained from healthy controls $(n=29)$, active ulcerative colitis $(n=49)$ and inactive ulcerative colitis $(\mathrm{n}=47)$ patients who underwent endoscopy examination at the Department of Gastroenterology, The First Affiliated Hospital of Zhengzhou University (Zhengzhou, China). A total of 82 frozen CRC tissues and their paired non-cancerous tissues were collected from The First Affiliated Hospital of Zhengzhou University. This research was approved by the ethics committee of The First Affiliated Hospital of Zhengzhou University and conducted in accordance with the Declaration of Helsinki. All patients have given written informed consent.

\section{Cell Culture}

A panel of human colon cancer cell lines, including SW620, LoVo, HT-29, Caco-2, HCT116 and SW480, as well as normal human colonic epithelial cell line CCD841, was purchased from the American Type Culture Collection (ATCC). These cells were all grown in DMEM medium replenished with 10\% FBS (Fisher Scientific, USA) and $1 \%$ penicillin/streptomycin. Cells were maintained under the standard condition $\left(37^{\circ} \mathrm{C}, 90 \%\right.$ humidity and $\left.5 \% \mathrm{CO}_{2}\right)$ and have been confirmed without mycoplasma contamination by MycoAlert Mycoplasma Detection Kit (Lonza). Cell culture dishes and plates were purchased from Hangzhou Xinyou Biotechnology Co., Ltd. (China). The inhibitors of JAK2/STAT3 signaling BP-1-102 and 
FLLL32 were purchased from Selleckchem (Shanghai, China).

\section{Plasmids Construction and Cell Transfection}

pLKO.1-SLC6A14 vector, pCDH-CMV-MCS-EF1 $\alpha$ SLC6A14 vector or their corresponding control vectors combined with packaging plasmids (ps-PAX2 and pMD2. G) were transfected into $293 \mathrm{~T}$ cells using Lipofectamine 3000 (Invitrogen, CA, USA). After 48h, lentiviruscontaining supernatants were collected, filtered and added into Caco-2 and HCT-116 cells accompanied by Polybrene $(10 \mu \mathrm{g} / \mathrm{mL})$. Thereafter, puromycin $(5 \mu \mathrm{g} / \mathrm{mL})$ was utilized to select stable SLC6A14-knockdown and SLC6A14overexpression CRC cells for 7 days.

\section{RNA Extraction and qRT-PCR}

Total RNA from tissues and cells were isolated by using RNAiso Plus reagent and quantified with a NanoDrop spectrophotometer. Then, $1000 \mathrm{ng}$ total RNA was reversetranscribed to generate first-strand cDNA by using the PrimeScript $^{\mathrm{TM}}$ RT Master Mix (TaKaRa, Japan) following the protocols of the manufacturer. Gene expression was analyzed by quantitative real-time PCR with SYBR Green Master Mix using a QuantStudio 7 Flex system. GAPDH and $\beta$-actin were used as reference genes and all experiments were conducted in triplicate. The relative mRNA expression was calculated using the $2^{-\Delta \Delta \mathrm{Ct}}$ method. ${ }^{18,19}$

\section{Immunohistochemistry, $\mathrm{Ki}-67$ and TUNEL Staining}

Immunohistochemistry staining of SLC6A14 from 45 CRC samples was carried out on paraffin-embedded colorectal tissue sections, which were deparaffinized in xylene, rehydrated in alcohol, and immersed in 3\% hydrogen peroxide to prevent endogenous peroxidase activity. Afterward, the slides were immunostained with the SLC6A14 antibody (ab254786) at $4^{\circ} \mathrm{C}$ overnight. After rinsing, the sections were then incubated with HRPconjugated secondary antibody for 1 hour at room temperature. The slides were stained with DAB solution and analyzed with a light microscope (Olympus, Japan). For the expression and localization of Ki-67, the colon slides of mouse were deparaffinized, rehydrated and treated according to a standard protocol. After incubating with primary antibody against $\mathrm{Ki}-67$ overnight at $4^{\circ} \mathrm{C}$ and corresponding secondary antibody, the sections were incubated with DAPI for $10 \mathrm{~min}$ to exhibit the location of the nucleus. Images of Ki-67 were captured by using an inverted fluorescence microscope. For TdT-mediated dUTP nick end labelling (TUNEL) staining of mouse colon tissues, paraffin sections of the biopsies were immersed in xylene to deparaffinize and a series of decreasing concentrations of alcohol to rehydrate. Then, the slides were treated with $3 \%$ hydrogen peroxide to block the activity of endogenous peroxidase, followed by the incubation with proteinase K. DNA fragmentation was analyzed by an In Situ Cell Death Detection Kit (Roche, Germany) according to the manufacturer's instruction. TUNEL-positive cells were observed and captured with an Olympus microscope.

\section{Western Blot}

Cells and colorectal mucosal tissues were lysed in RIPA buffer containing protease and phosphatase inhibitors (Beyotime, China). Total protein concentrations were measured by using a BCA protein assay kit (Thermo Scientific, USA). Equal amounts of cell protein samples $(20 \mu \mathrm{g})$ and human tissue samples $(50 \mu \mathrm{g})$ were loaded onto an SDSPAGE gel and electrophoresed. Then, the separated protein samples were transferred onto PVDF membranes (Millipore, USA) and the membranes were blocked with 5\% BSA in TBST buffer at room temperature for 1 hour and incubated with appropriate primary antibodies at $4{ }^{\circ} \mathrm{C}$ overnight. After washing the membranes with TBST for three times, the membranes were incubated with corresponding HRPconjugated secondary antibodies at room temperature for 1 hour. Finally, ECL chemiluminescence reagents were added to the membranes to magnify immunoreactive signals, which were subsequently detected and visualized by a BioRad detection system. The antibodies used in this study were listed as follows: SLC6A14 (Abcam, ab99102), p-JAK2 (Tyr1007/1008, Cell Signaling Technology, Cat\#3771), JAK2 (Cell Signaling Technology, Cat\#3230), p-STAT3 (Tyr705, Cell Signaling Technology, Cat\#9131), STAT3 (Cell Signaling Technology, Cat\#30,835), $\beta$-actin (BIOSS, Cat\#bs-0061R).

\section{Cell Proliferation and Colony Formation Assay}

Cell growth rate was detected by using commercial cell counting kit- 8 reagent (Donjindo, Japan). CRC cells at a density of $1 \times 10^{3}$ per well were seeded into a 96-well plate with five repeats. After 24 hours' incubation, cells in 
each well were added with $10 \mu \mathrm{L}$ CCK-8 solution and 90 $\mu \mathrm{L}$ cell culture medium and incubated for another 2 hours at $37^{\circ} \mathrm{C}$. Finally, the absorbance at $450 \mathrm{~nm}$ was detected with a multifunctional microplate reader for consecutive five days. For colony-formation experiments, about 500 cells resuspended in the complete medium were seeded into six-well plates and cultured in the incubator for consecutive 14 days. The medium change was conducted at three-day intervals. Cells were fixed with $4 \%$ paraformaldehyde, washed with PBS and stained with crystal violet. Images of colonies were captured for further analysis.

\section{Cell Apoptosis Analysis by Flow Cytometry}

Annexin-V/propidium iodide (PI) staining is a typical flow cytometric method for detecting cell apoptosis. ${ }^{20}$ Cells at a density of $1 \times 10^{6}$ were collected and resuspended in 500 $\mu \mathrm{L}$ binding buffer containing $5 \mu \mathrm{L}$ Annexin $\mathrm{V}$ and $10 \mu \mathrm{L}$ PI. After incubation in the dark for 15 minutes, samples were analyzed immediately with a FACSCanto flow cytometer (BD Bioscience, USA).

\section{Cell Migration and Invasion Assay}

Matrigel for invasion assay was thawed slowly on the ice at $4^{\circ} \mathrm{C}$ overnight. Chambers inserts for invasion assay were coated with a layer of diluted Matrigel and dried at $37^{\circ} \mathrm{C}$. For migration and invasion assays, CRC cells were grown and treated in the same manner as previously described. $^{21}$ In brief, $1 \times 10^{5}$ CRC cells per well resuspended in serum-free media were plated into the upper chamber and 20\% FBS-containing complete medium was added into the bottom room as a chemoattractant. After incubation at $37^{\circ} \mathrm{C}$ for 24 hours, the migrated cells were fixed with $4 \%$ paraformaldehyde, and non-migratory cells in the upper chamber were removed by a sterile cotton swab. Finally, the attached migratory cells were stained with $0.3 \%$ crystal violet and quantified with a Nikon inverted microscope.

\section{Animal Studies and AOM/DSS-Induced Colorectal Carcinogenesis Model}

All animal experimentation was conducted under the approval of the Laboratory Animal Care and Welfare Committee of Zhengzhou University and the procedures were complied with the Animal Welfare Act and the Guide for the Care and Use of Laboratory Animals approved by the Chinese Association for Laboratory Animal Sciences.
For the induction of Azoxymethane/Dextran sulfate sodium (AOM/DSS) model, wild-type C57BL/6 male mice (6-8 weeks old) were administrated with a single dose of $12.5 \mathrm{mg} / \mathrm{kg}$ AOM (Sigma-Aldrich, USA) via intraperitoneal injection on the first day and recovered for next 5 days. Then, the mice were treated with two cycles of $2.5 \%$ dextran sulfate sodium (DSS, MP Biochemicals, USA) for 5 days and regular drinking water for 14 days. After the final cycle of $2 \%$ DSS for 5 days and recovered for a month, the mice were euthanized. The colons were dissected longitudinally, polyps and tumors were blindly assessed and counted. During the whole period, mice were weighed and monitored every three days for bodyweight changes and other symptoms to evaluate CRC progression. For in vivo tumor xenograft model, female Balb/c nude mice (6-week-old) were purchased from Shanghai SLAC Laboratory Animal and maintained with free access to diet or water. The mice were divided into two groups at random and injected subcutaneously with equal amount of OE-SLC6A14 and pCDH cells $\left(2 \times 10^{6}\right.$ cells $)$. Tumor size was checked with a caliper once a week and recorded. After a month, the mice were euthanized, and tumors were isolated.

\section{Statistical Analysis}

Experimental data were analyzed by GraphPad Prism 8.0 software and presented as mean \pm standard deviation (SD). Differences between groups were analyzed by paired Student's $t$-test, unpaired Student's $t$-test. Chi-square test was used to analyze the relationship between SLC6A14 expression and clinicopathological parameters. Clinical correlations in human colorectal tissues were performed using Pearson's correlation coefficient. Overall survival curves were analyzed by using Kaplan-Meier method. Data for statistical analysis were from at least three representative independent experiments. $* \mathrm{P}<0.05, * * \mathrm{P}<0.01$, and $* * * \mathrm{P}<0.001$ were considered as statistical significance.

\section{Results}

\section{SLC6AI4 is Upregulated in Active UC and Colorectal Cancer}

By analyzing three public GEO datasets, we found that SLC6A14 mRNA expression showed a remarkable increase in active ulcerative colitis (UC) and colorectal cancer tissues compared with normal colorectal samples (Figure 1A). We further performed qRT-PCR in our cohort to evaluate the 
SLC6A14 expression level in patients with CRC or colitis. As shown in Figure 1B, we noticed an elevated SLC6A14 mRNA level in the inflamed mucosa of active UC patients $(n=49)$ compared to samples of healthy controls $(n=29)$ and inactive UC $(\mathrm{n}=47)$ patients. Moreover, SLC6A14 was dramatically upregulated in CRC tissues $(\mathrm{n}=82)$ in comparison with matched adjacent normal counterparts (Figure 1C). Next, we evaluated SLC6A14 protein levels in 12 paired CRC tumor tissues and matched adjacent normal tissues by Western blot, and a similar trend was discovered (Figure 1D).
We further investigated the correlation between SLC6A14 and clinicopathological characteristics in 82 CRC patients, and CRC patients were stratified as high and low group according to the median level of SLC6A14 expression. Our results showed that high SLC6A14 expression was closely correlated with lymph node metastasis and advanced TNM stage of CRC patients (Figure S1A and Table 1). Besides, immunostaining was carried out using samples from another cohort of 45 CRC patients and representative SLC6A14 immunostaining images are exhibited in Figure 1E. Based
A
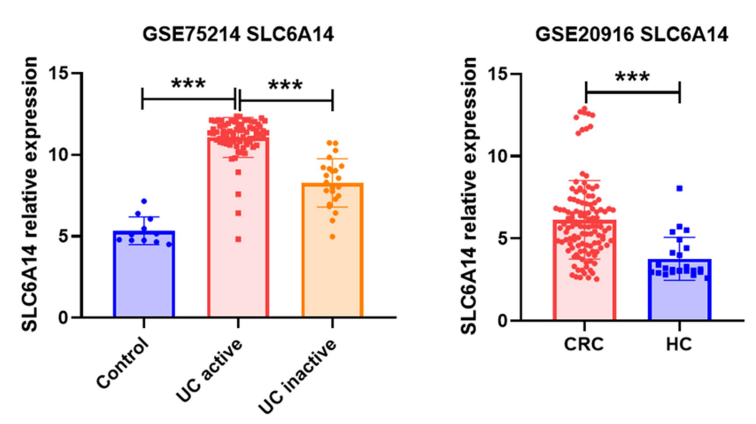

C
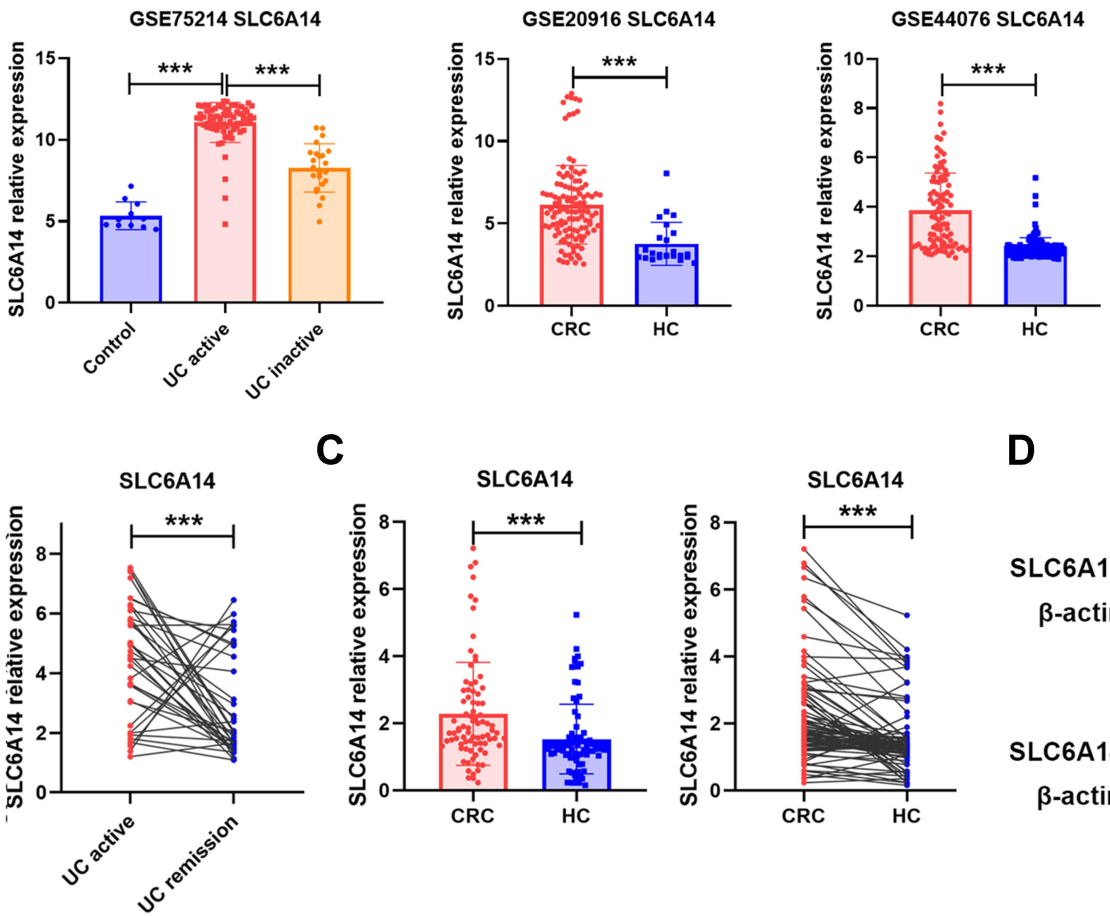

B

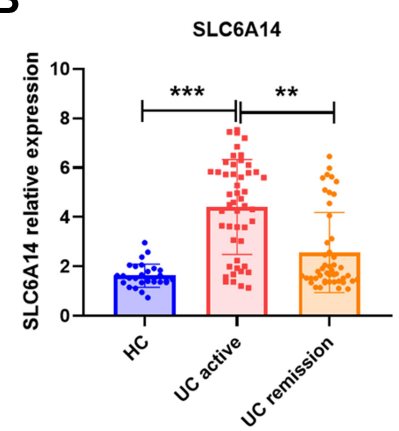

D
E
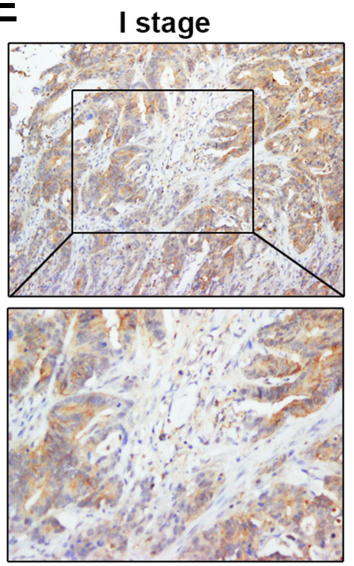

IIA stage
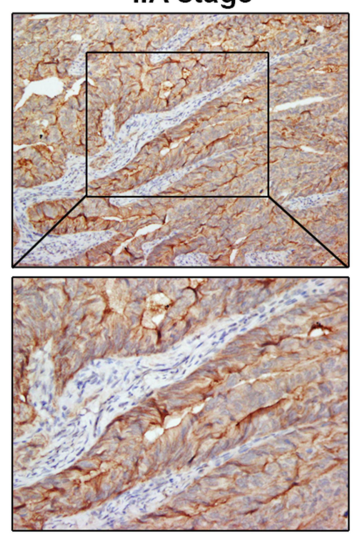

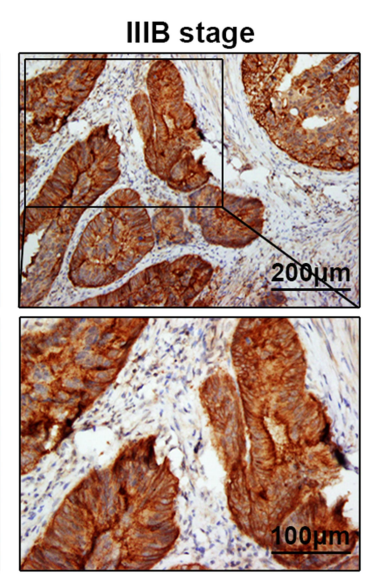

\section{$\mathbf{F}$}

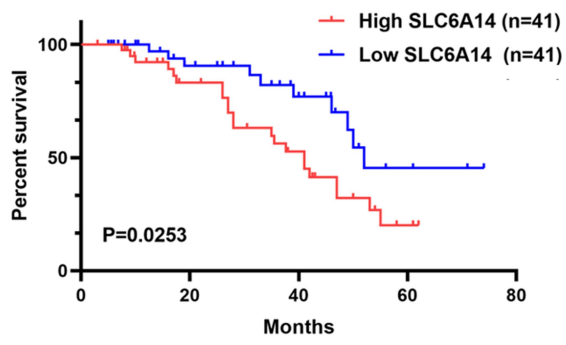

Figure I SLC6AI4 is increased in active UC and CRC tumor tissues. (A) Bioinformatics analysis of SLC6AI4 expression in inflamed mucosal tissues of active UC patients and tumor tissues of CRC patients based on GEO datasets. (B) RT-PCR analysis of SLC6AI4 mRNA level in different stages of UC patients. (C) RT-PCR analysis of SLC6AI4 expression in CRC tissues and paired non-cancerous tissues. (D) Immunoblot analysis of SLC6AI4 protein level in 12 paired of human CRC tissues and adjacent normal colorectal samples. (E) Immunohistochemical analysis of differential SLC6AI4 expression in CRC patients. (F) Kaplan-Meier analysis of the association between SLC6AI4 expression and overall survival in CRC patients. $* * \mathrm{P}<0.01$ and $* * * \mathrm{P}<0.001$ were considered as significant differences. Bars in all graphs represented mean \pm S.D. 
on the mRNA level of SLC6A14, Kaplan-Meier survival analysis showed that CRC patients with high SLC6A14 expression had poor overall survival rate (Figure 1F). Taken together, these results demonstrated that SLC6A14 might serve as a vital oncogene during the progression of CRC.

\section{Pharmacologic Blockade of SLC6AI4 Protects Against Intestinal Colitis-Associated Tumorigenesis in vivo}

To investigate the functional role of SLC6A14 in the pathogenesis of CRC, we constructed azoxymethane and dextran sulfate sodium (AOM-DSS)-induced CRC models by administrated mice with AOM and three cycles of DSS. In addition, we used a non-transportable blocker $\alpha$-MT to achieve pharmacologic blockade of SLC6A14 function in vivo. Compared with AOM-DSS treated mice, extra

Table I Correlation Between mRNA Expression of SLC6AI4 and Clinical Characteristics in 82 CRC Patients

\begin{tabular}{|c|c|c|c|c|}
\hline \multirow[t]{2}{*}{ Variables } & \multirow{2}{*}{$\begin{array}{l}\text { No. of } \\
\text { Patients }\end{array}$} & \multicolumn{2}{|c|}{ SLC6A14 Expression } & \multirow[t]{2}{*}{$P$-value } \\
\hline & & $\begin{array}{l}\text { High } \\
\text { Group } \\
(n=4 I)\end{array}$ & $\begin{array}{l}\text { Low } \\
\text { Group } \\
(n=4 I)\end{array}$ & \\
\hline \multicolumn{5}{|l|}{ Gender } \\
\hline Male & 39 & 20 & 19 & 0.8250 \\
\hline Female & 43 & 21 & 22 & \\
\hline \multicolumn{5}{|l|}{ Age } \\
\hline$<50$ & 40 & 23 & 17 & 0.2692 \\
\hline$\geq 50$ & 42 & 18 & 24 & \\
\hline \multicolumn{5}{|l|}{ Tumor grade } \\
\hline $\mathrm{Gl}+\mathrm{G} 2$ & 45 & 23 & 22 & 0.8244 \\
\hline $\mathrm{G} 3+\mathrm{G} 4$ & 37 & 18 & 19 & \\
\hline \multicolumn{5}{|l|}{ Tumor invasion } \\
\hline $\mathrm{TI}+\mathrm{T} 2$ & 39 & 17 & 22 & 0.3766 \\
\hline $\mathrm{T} 3+\mathrm{T} 4$ & 43 & 24 & 19 & \\
\hline \multicolumn{5}{|l|}{ Size (cm) } \\
\hline$<5$ & 35 & 14 & 21 & 0.1800 \\
\hline$\geq 5$ & 47 & 27 & 20 & \\
\hline \multicolumn{5}{|l|}{$\begin{array}{l}\text { Lymph node } \\
\text { status }\end{array}$} \\
\hline Negative & 34 & 10 & 24 & $0.0033^{* *}$ \\
\hline Positive & 48 & 31 & 17 & \\
\hline \multicolumn{5}{|l|}{ TNM stage } \\
\hline$|+| \mid$ & 36 & 12 & 24 & $0.0139 *$ \\
\hline III+IV & 46 & 29 & 17 & \\
\hline
\end{tabular}

Notes: $* \mathrm{P}<0.05 .{ }^{*} * \mathrm{P}<0.01$. pharmacologic blockade of SLC6A14 with $\alpha$-MT conspicuously reduced the weight loss and expression of SLC6A14 in colon tissues of mice (Figure 2A and Figure S2A). However, there was no significant difference in the colon length of mice between AOM-DSS group and AOM-DSS plus $\alpha$-MT treated group (Figure 2B). Moreover, inhibiting SLC6A14 with $\alpha$-MT markedly diminished the numbers of macroscopic tumor burden in the colon compared with AOM-DSS treated mice (Figure 2C). To evaluate the effects of $\alpha$-MT on proliferation and apoptosis properties, intestinal swiss roll was subjected to Ki-67 and TUNEL staining. As shown in Figure 2D and E, accelerated proliferation and attenuated apoptosis were observed in AOM-DSS treated mice, whereas the percentage of Ki-67 positive cells in $\alpha$-MT treated mice was notably reduced, and the apoptosis rate in $\alpha$-MT treated mice was prominently increased (Figure S2B and $\underline{\mathrm{C}}$ ). Collectively, these findings indicated that SLC6A14 promoted CRC tumorigenesis by affecting growth and apoptosis of tumor cells in vivo.

\section{SLC6AI4 Expedites CRC Cell Proliferation and Suppresses Cell Apoptosis in vitro}

We investigated the SLC6A14 expression in a panel of human CRC cell lines and normal colonic epithelial cell line CCD841 by immunoblotting and qRT-PCR. Notably, SLC6A14 was significantly upregulated in the majority of cancer cell lines, with Caco-2 had the highest level, while HCT-116 showed the lowest level (Figure 3A). To figure out the potential function of SLC6A14 in CRC progression, we established stably overexpressing SLC6A14 in HCT-116 cells and silenced SLC6A14 in Caco-2 cells by target shRNA. The overexpression or knockdown efficiency was confirmed by qRT-PCR and Western blot (Figure 3B and C). Next, we conducted CCK-8 assay to evaluate the effect of SLC6A14 on cell proliferation, and the results showed that overexpression of SLC6A14 evidently accelerated cell proliferation in HCT-116 cells. In contrast, silencing of SLC6A14 decelerated cell growth in Caco-2 cells (Figure 3D). Similarly, results from colonyformation assays revealed that knockdown of SLC6A14 prominently restrained clonogenicity abilities in Caco-2 cells, while overexpression of SLC6A14 enhanced the colony formation in HCT-116 cells (Figure 3E). In addition, we found the $\mathrm{H}_{2} \mathrm{O}_{2}$ induced-apoptosis rate was decreased in OE-SLC6A14 cells, while knockdown of 
A

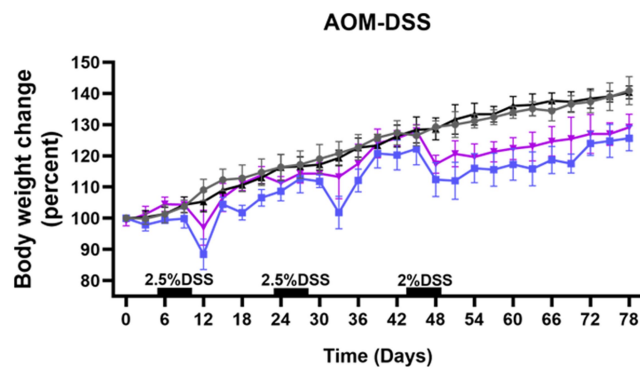

D

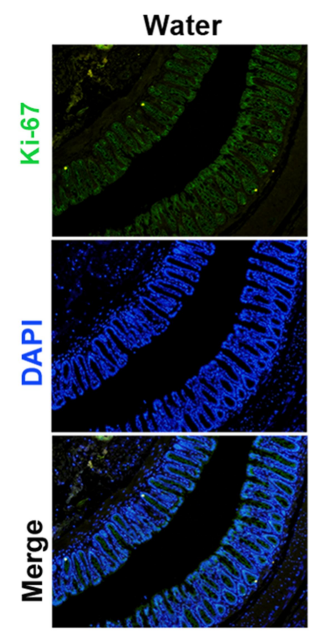

AOM-DSS

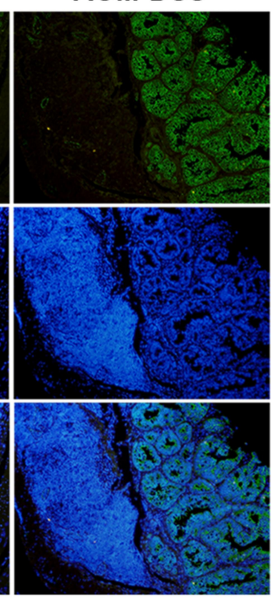

$\rightarrow$ Water

- AOM-DSS

- $\alpha-M T$

- AOM-DSS+a-MT
B

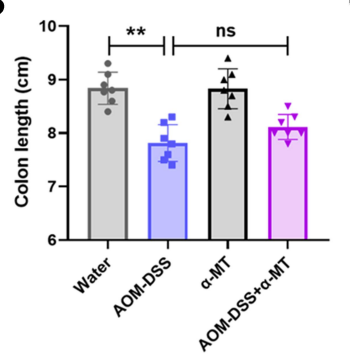

C

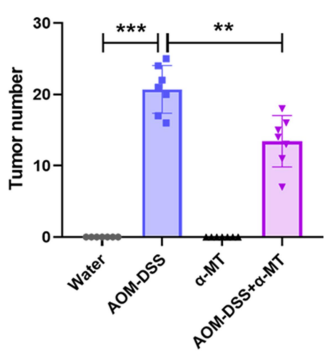

E

\begin{abstract}
AOM-DSS+ $-\alpha-M T$
\end{abstract}

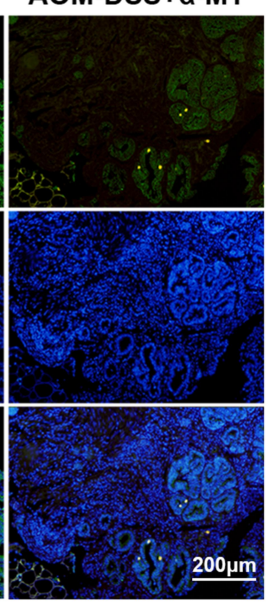

Water

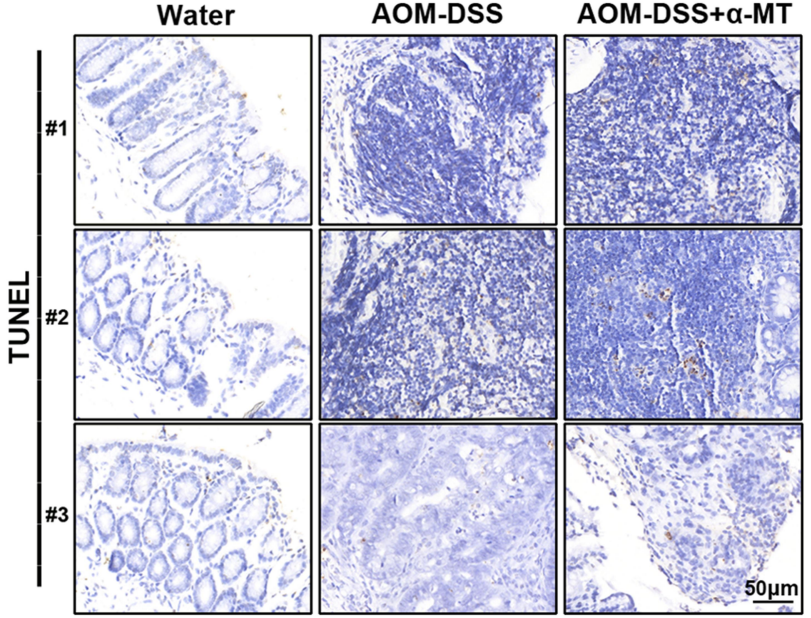

Figure 2 Pharmacologic blockade of SLC6AI4 with $\alpha$-MT suppresses colitis-associated tumorigenesis in vivo. (A) Bodyweight changes in the control, AOM-DSS, $\alpha$-MT and AOM-DSS plus $\alpha$-MT group mice during the whole period. (B) Colon length of different treated mice was evaluated. (C) Tumor numbers in the colon of different treated mice were determined. (D) Proliferation was detected by Ki-67 immunofluorescence staining. (E) Apoptosis was determined by TUNEL staining. Bars in all graphs represented mean \pm S.D., $\mathrm{n}=7$ per group; $* * \mathrm{P}<0.01$ and $* * * \mathrm{P}<0.00 \mathrm{I}$.

SLC6A14 observably reversed this trend, as evidenced by flow cytometry (Figure 3F). Additionally, pharmacologic blockade of SLC6A14 function with a non-transportable blocker $\alpha$-MT effectively inhibited cell proliferation, colony formation and increased cell apoptosis in Caco-2 cells (Figure 3D-F). These data established a crucial role of SLC6A14 in modulating cell growth and apoptosis of CRC cells in vitro.

\section{SLC6AI4 Promotes Migration of CRC}

\section{Cells in vitro}

We further evaluated the effects of SLC6A14 overexpression or knockdown on the migratory and invasive capacities of CRC cells by transwell migration and invasion assays. As manifested in Figure 4A, overexpression of SLC6A14 in HCT-116 cells markedly facilitated its mobility compared with control cells, and a consistent result was obtained from invasion assay. However, inhibition of SLC6A14 in Caco-2 cells notably impaired their ability to migrate or invade through the transwell chambers in comparison with the control cells (Figure 4B). Therefore, these results demonstrated that SLC6A14 had its biological function to accelerate the migration and invasion abilities of CRC cells.

\section{SLC6A 4 Promotes the Malignant Phenotypes of CRC Cells via JAK2/STAT3 Signaling}

To illustrate the underlying mechanism of SLC6A14 in CRC tumorigenesis, we undertook the GSEA enrichment analysis using TCGA datasets. Intriguingly, we found that JAK2/STAT3 signaling was shown as one of the most prominently altered pathways in CRC patients with high SLC6A14 expression (Figure 5A and Figure S3). Meanwhile, GSE20916 dataset also verified the increasement of JAK2 and STAT3 expression in CRC tumor tissues (Figure 5B). And the elevated SLC6A14 in CRC tissues was tightly correlated with the elevated JAK2 (Spearman's rank correlation coefficient, $\mathrm{R}=0.4980$, $\mathrm{P}<0.0001$ ) and STAT3 (Spearman's rank correlation 
A
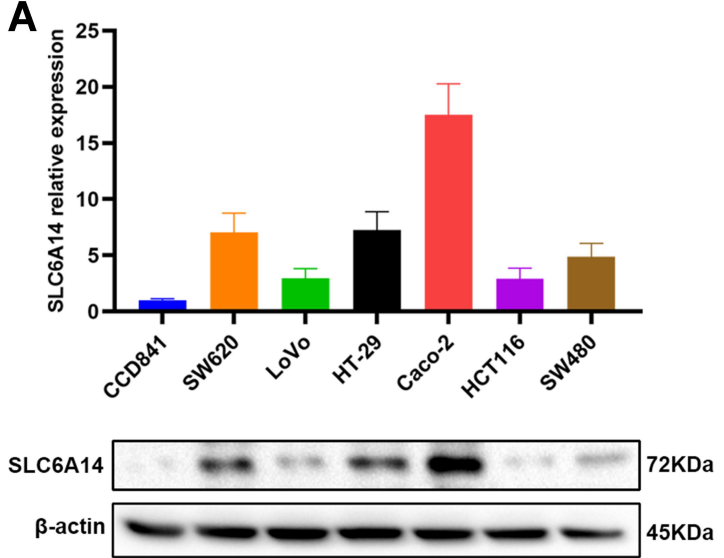

B
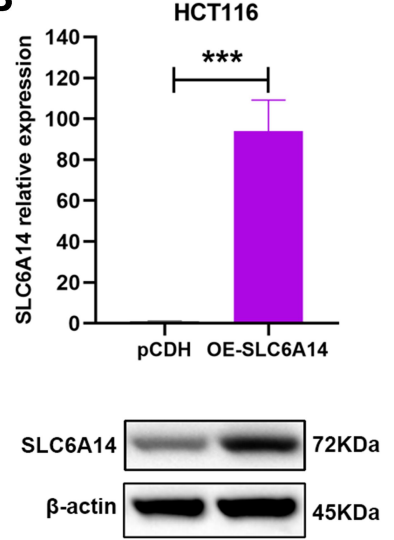

Caco-2

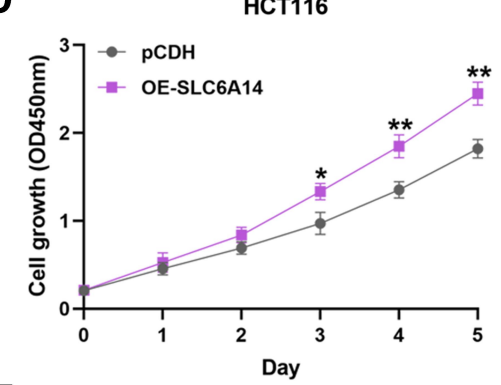

E

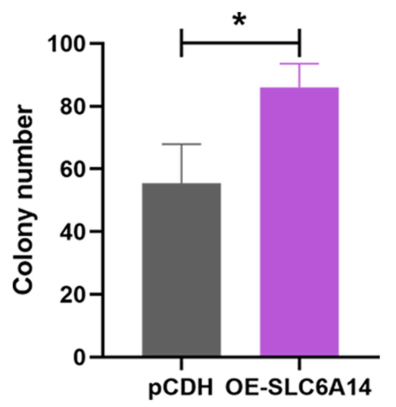

F

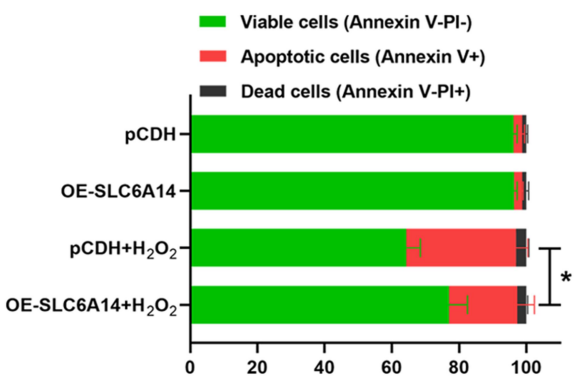

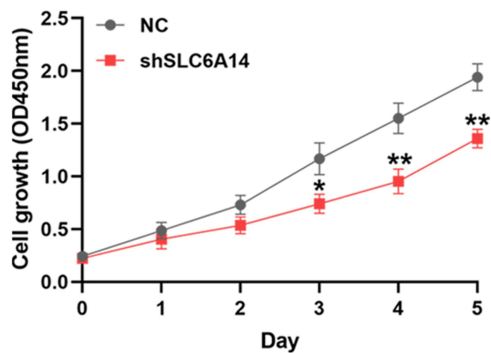
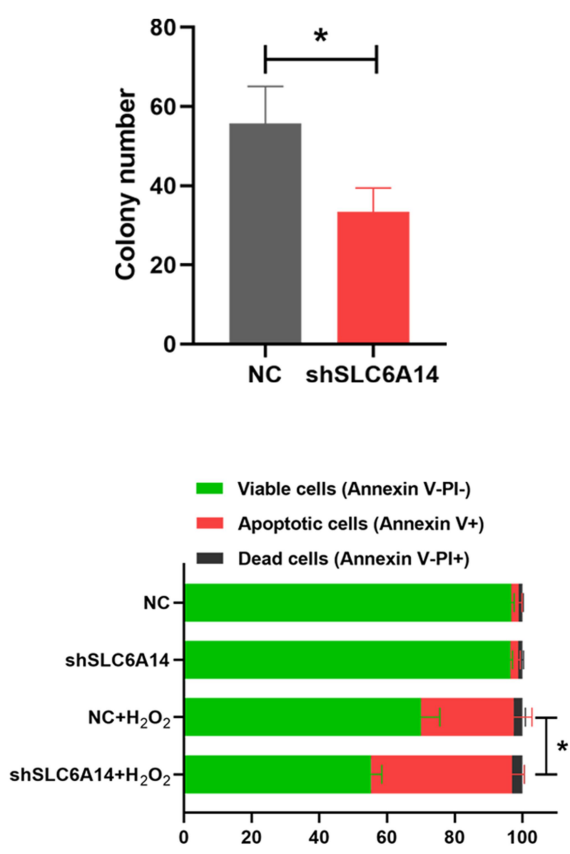

C
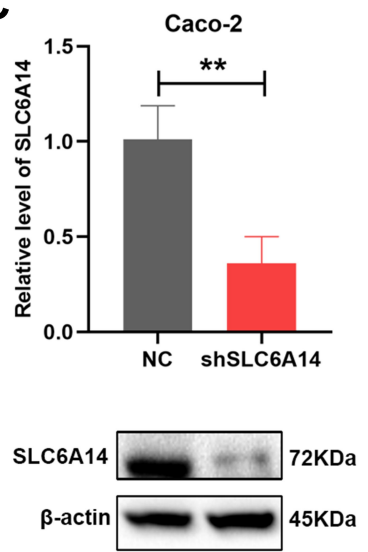

Caco-2
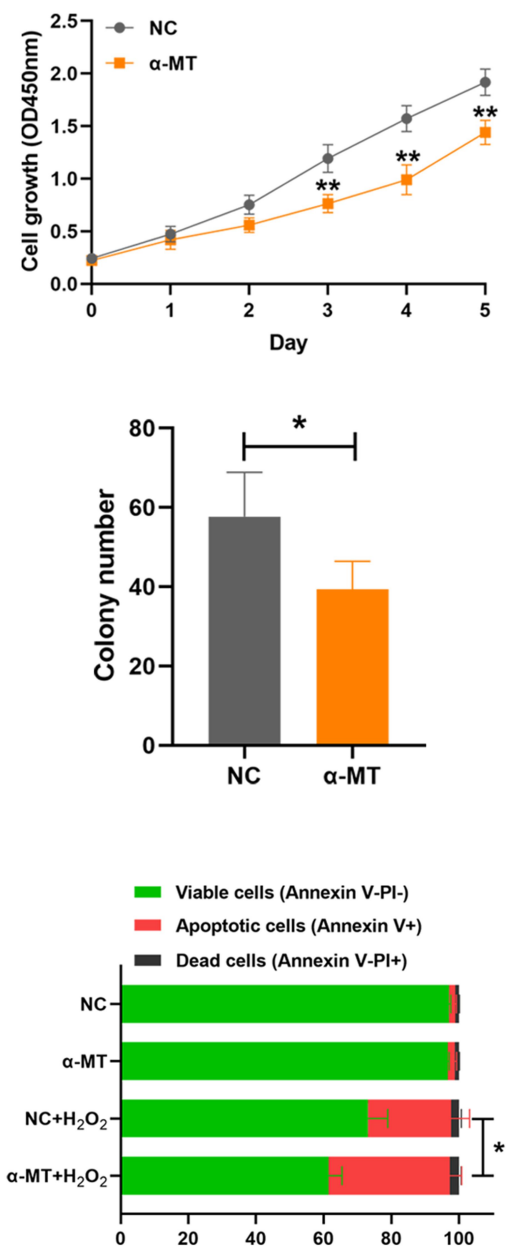

Figure 3 SLC6AI4 facilitates cell proliferation and inhibits cell apoptosis in vitro. (A) SLC6AI4 expression level was detected in a series of CRC cell lines and normal colonic epithelial cell line by qRT-PCR and immunoblot. (B, C) The overexpression and knockdown efficiency of SLC6AI4 were examined by qRT-PCR and immunoblot. (D) CCK-8 assay was performed to access the effects of modulating SLC6AI4 on cell proliferation of CRC cells. (E) Colony-forming assays in SLC6AI4 overexpression and depletion CRC cells were conducted. (F) The percentage of cell apoptosis after regulating SLC6AI 4 expression was determined by flow cytometric analysis. $* \mathrm{P}<0.05$, $* * \mathrm{P}<$ 0.01 , and ${ }^{* * *} \mathrm{P}<0.00 \mathrm{I}$. Bars in all graphs represented mean \pm S.D.

coefficient, $\mathrm{R}=0.4654, \mathrm{P}<0.0001$ ) level (Figure $5 \mathrm{C}$ ). We also evaluated the expression of JAK2 and STAT3 and their correlation with SLC6A14 in our cohort of 82 CRC tissues and paired normal tissues. Similarly, there were significant positive correlations between the upregulated SLC6A14 and JAK2 (Spearman's rank correlation 
A
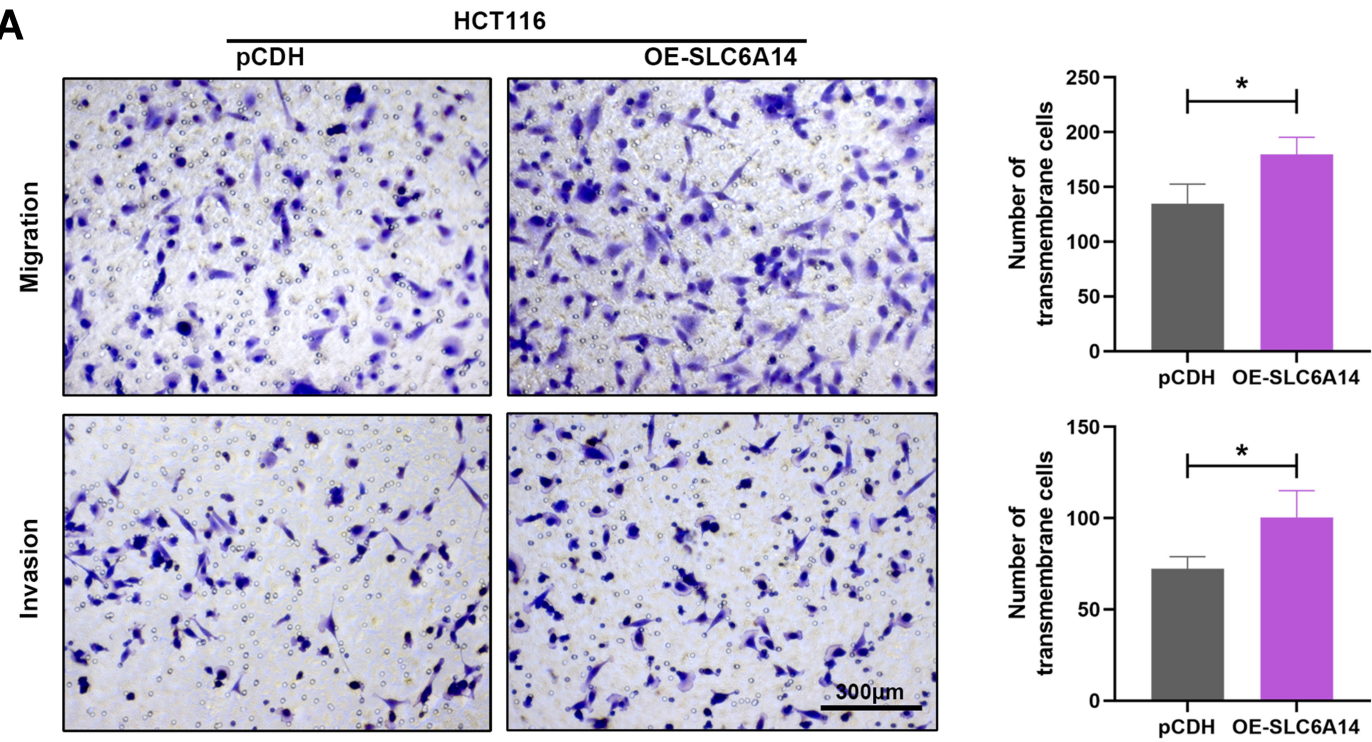

$\mathbf{B}$

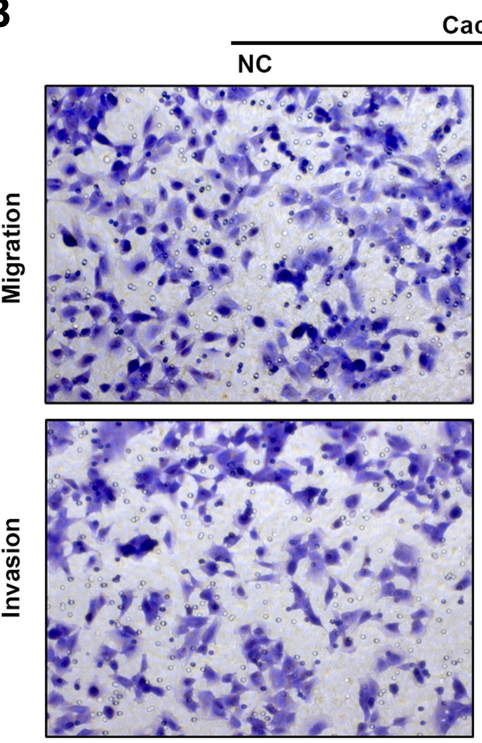

Caco-2
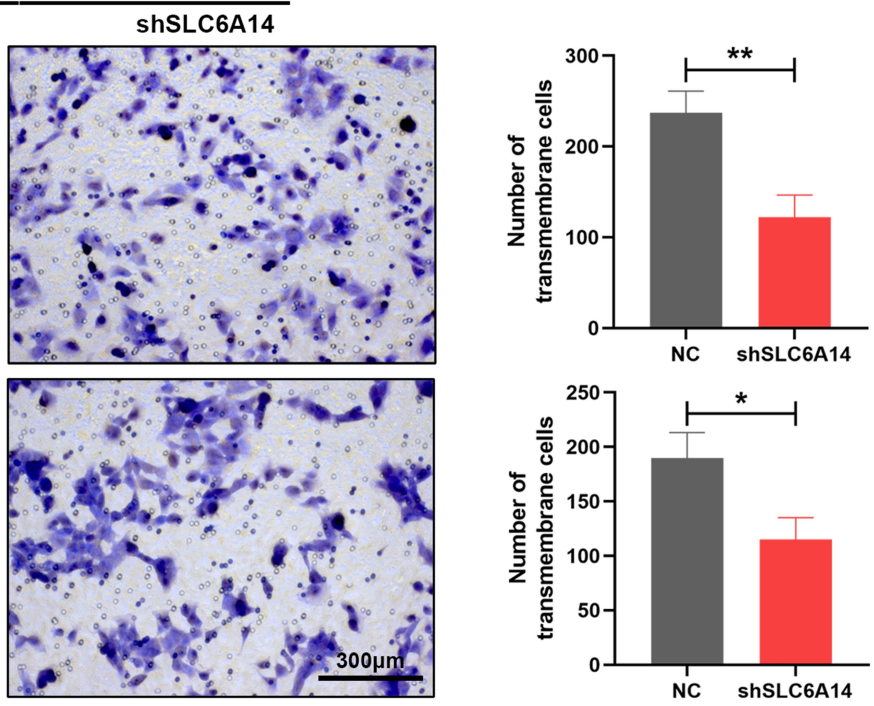

Figure 4 SLC6AI4 accelerates migration and invasion abilities of CRC cells in vitro. (A) Transwell migration and invasion assays to determine the effects of SLC6AI4 overexpression on the migratory and invasive abilities of HCT-II6 cells. (B) Transwell migration and invasion assays to detect the effects of SLC6AI4 knockdown on the mobility and invasiveness of Caco- 2 cells. $* \mathrm{P}<0.05$, $* * \mathrm{P}<0.01$. Bars in all graphs represented mean \pm S.D.

coefficient, $\mathrm{R}=0.3461, \mathrm{P}=0014$ ), as well as STAT3 (Spearman's rank correlation coefficient, $\mathrm{R}=0.3489$, $\mathrm{P}=0013$ ) (Figure 5D and E). Besides, we determined the protein levels of total and phosphorylation JAK and STAT3 after modulating the expression of SLC6A14 in CRC cells. As shown in Figure 5F, SLC6A14 dramatically increased the protein level of phosphorylated JAK and STAT3, whereas knockdown of SLC6A14 showed opposite effects (Figure 5F). We further utilized inhibitors specifically targeting JAK2/STAT3 pathway (BP-1-102 and FLLL32) to investigate the involvement of JAK2/ STAT3 signaling on SLC6A14-mediated malignant phenotypes in CRC. Strikingly, BP-1-102 and FLLL32 robustly abolished the exacerbation of cell proliferation, colony formation, migration and invasion mediated by exogenous SLC6A14 in HCT-116 cells (Figure 5G). Collectively, these data suggested that SLC6A14 promoted malignant cell growth and migration through JAK2/STAT3 pathway in CRC cells.

\section{Overexpression of SLC6AI4 Facilitates}

\section{Tumor Growth in vivo}

Finally, we utilized immunodeficient Balb/c nude mice to explore the role of SLC6A14 on CRC tumorigenesis in vivo. 
A

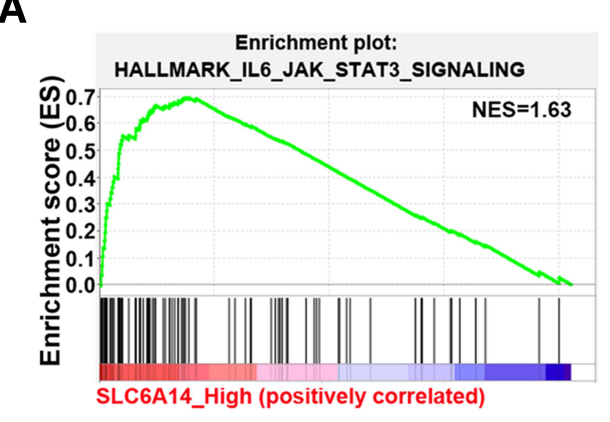

C
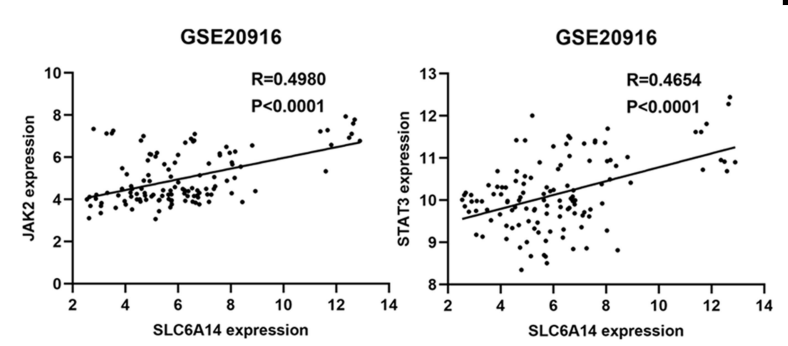

E
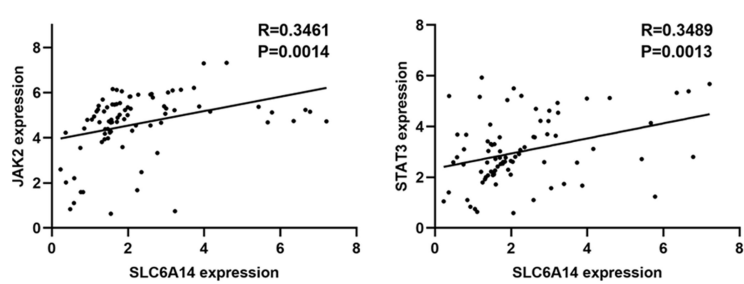

G

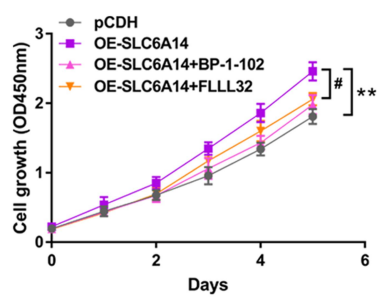

B

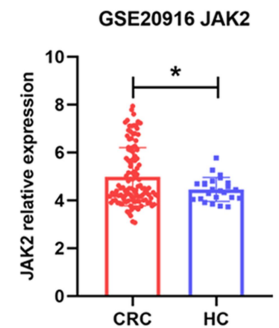

D
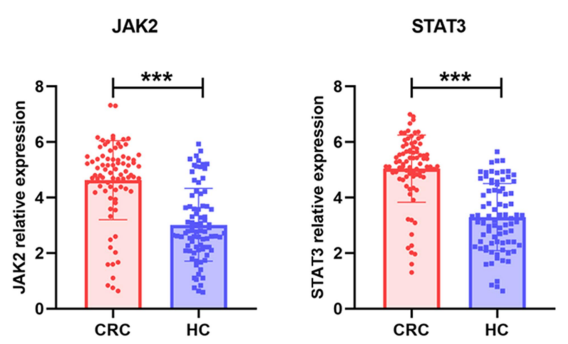

F
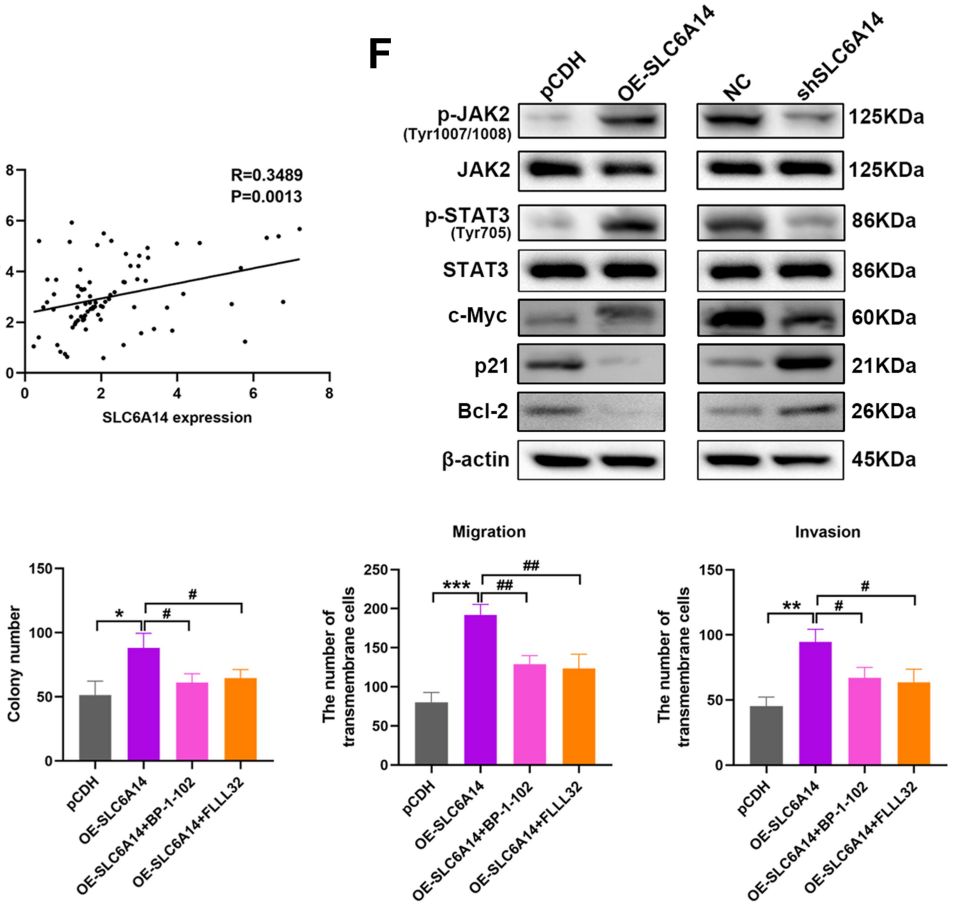

Figure 5 SLC6A I4-induced malignant phenotypes is mediated by JAK2/STAT3 signaling. (A) GSEA enrichment analysis in CRC patients with SLC6AI4 high expression to explore potential pathways correlated with SLC6AI4. (B) Bioinformatics analysis of JAK2 and STAT3 expression in the tumor tissues of CRC patients based on GEO datasets. (C) Correlation analysis between SLC6AI4 and JAK2/STAT3 signaling based on GSE20916. (D) RT-PCR analysis of JAK2 and STAT3 expression in CRC tissues and paired non-cancerous tissues. (E) Correlation analysis between SLC6AI4 and JAK2/STAT3 signaling based on a cohort of 82 CRC patients. (F) The effects of SLC6AI4 on the protein levels of JAK2/STAT3 signaling were determined by Western blot. (G) JAK2/STAT3 pathway-specific inhibitors BP-I - 102 and FLLL32 were utilized to explore the effects on the malignant phenotypes in SLC6AI4-overexpressing HCT-II 6 cells. Bars in all graphs represented mean \pm S.D. Significant differences were shown by $* P<0.05$, **P $<0.01$, and ***P $<0.001$. Significant differences from OE-SLC6Al4 cells were shown by ${ }^{\#} \mathrm{P}<0.05,{ }^{\#} \mathrm{P}<0.01$.

The mice were randomly divided into two groups, equal amounts of pCDH and OE-SLC6A14 HCT116 cells were injected subcutaneously into the mice, respectively. Interestingly, overexpression of SLC6A14 significantly facilitated the tumor growth in vivo compared with the control group mice (Figure 6A). Notably, there was a markedly increase in the final tumor size and weight in SLC6A14overexpressed group at the termination of the experiment (Figure 6B and C). Moreover, immunohistochemistry staining further revealed that there was a positive association between 
A

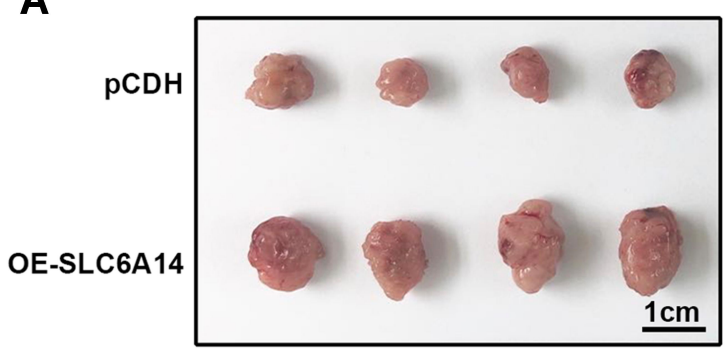

D
B

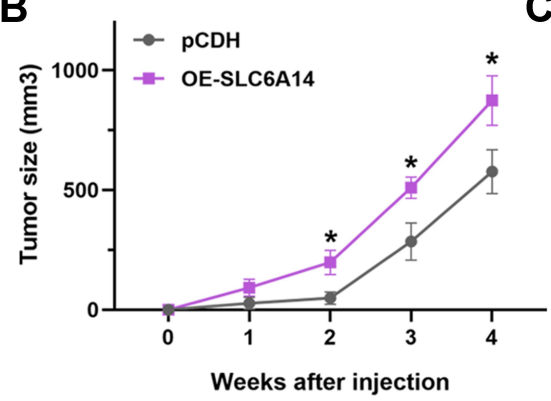

C

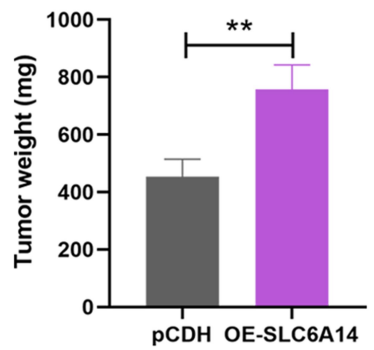

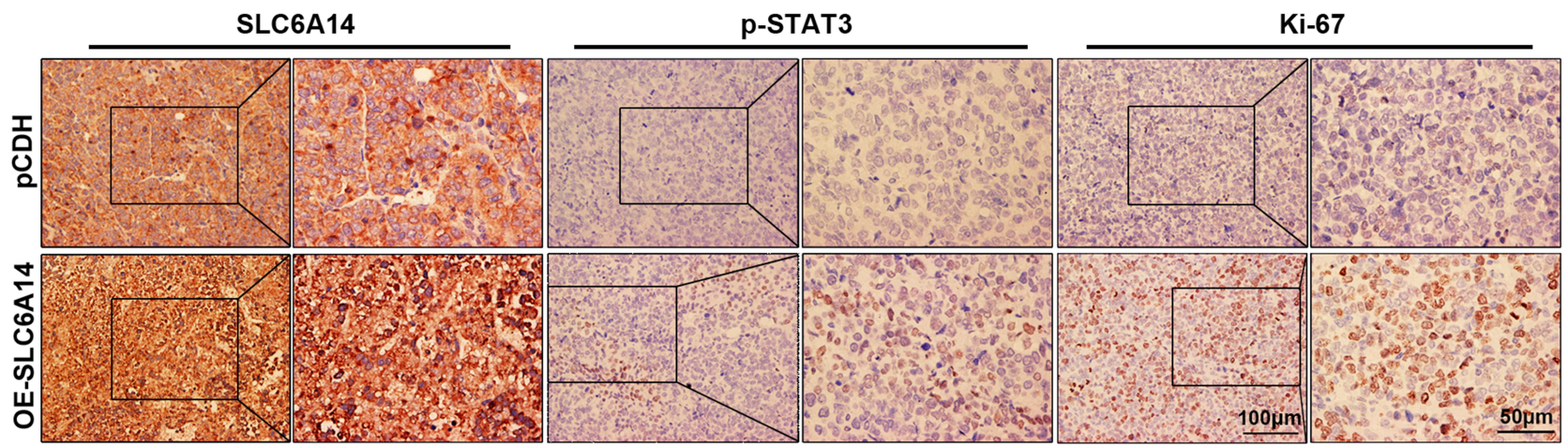

Figure 6 SLC6A 4 promotes xenograft tumor growth in vivo. (A) Images of tumors from the control group and SLC6AI4 overexpression group. (B) Tumor growth curves in two groups. (C) The differences in tumor weight between two different groups. (D) Immunohistochemical staining of SLC6AI4 and JAK2/STAT3 signaling expression in tumor tissues. Significant differences were shown by $* \mathrm{P}<0.05$, $* * \mathrm{P}<0.01$.

the upregulated SLC6A14 and activated STAT3 and Ki-67 level (Figure 6D). In summary, the above in vivo and in vitro data corroborated the oncogenic role of SLC6A14 in CRC progression.

\section{Discussion}

In living organisms, amino acid transporters localized at the cellular plasma membrane and intracellular organelle compartments, facilitating the uptake and exchange of various amino acids across membranes as essential materials for some vital fundamental biological functions such as energy metastasis and nitrogen balance. ${ }^{22}$ To date, the SLC superfamily is the largest family of membrane transport proteins in mammalian cells. ${ }^{23,24}$ In tumor cells, the imbalance of amino acid transporters, characterized by changes of intracellular amino acid levels will result in the dysregulation of cellular metabolic reprogramming and the pathogenesis of cancers. $^{25,26}$ Indeed, a series of neutral amino acid transporters including SLC1A5, SLC6A14, SLC7A5 and SLC7A11 are likely to participate in several malignant tumors to meet the considerable demands of amino acids for cancer cell nutrition. ${ }^{6-10}$ Increasing studies have shown that SLC1A5 mediated the uptake of glutamine, which is an essential amino acid equipped with multiple functions such as nucleotide synthesis, protein synthesis and mTORC1 signaling pathway activation for rapid tumor cell proliferation. ${ }^{27}$ In human breast cancer, a pharmacological inhibitor of SLC1A5 transport function prominently decreased the glutamine uptake, subsequently led to the inhibition of mTORC1 signaling, cell growth and cell cycle process. ${ }^{28}$ And the lower expression of SLC1A5 endowed a better survival advantage in the xenografted mice. In addition, SLC7A5 not only supported mTORC1 activity through promoting the efficient uptake of leucine into cells but also enhanced the activation of c-Myc and EZH2 related pathway in tumor cells. ${ }^{29}$ In some subtypes of human breast cancer, high expression of SLC7A5 was strongly associated with higher grade, larger tumor volume, which may serve as a promising therapeutic target. ${ }^{7}$ Another transporter that has attracted increasing attention regarding tumor growth is SLC7A11, which protected tumor cells against ferroptosis-mediated cell death when suffered from oxidative stress and nitrosative stress. ${ }^{30}$

SLC6A14 is a high-capacity and concentrative amino acid transporter. Leucine, as a substrate of SLC6A14, plays a crucial role in activating mTOR signaling pathway in tumor cells. Additionally, SLC6A14 also participated in the cellular glutathione synthesis process, as glycine is 
another substrate of SLC6A14. Therefore, the functional role of SLC6A14 in tumor cells is to guarantee amino acid nutrition, mTOR activation and prevent from oxidative stress to ensure quickly cell proliferation. By analyzing the public GEO datasets and our cohort of patients with CRC or colitis, we found that SLC6A14 was significantly upregulated in active UC and CRC, and high SLC6A14 expression was closely correlated with lymph node metastasis and advanced TNM stage of CRC patients, indicating that SLC6A14 might function as a vital oncogene during the progression of CRC. In pancreatic cancer, a pharmacological blocker of SLC6A14 with $\alpha$ methyltryptophan $(\alpha-M T)$ significantly interfered with amino acid nutrition and inhibited cells proliferation both in vitro and in vivo. ${ }^{10}$ In estrogen receptor-positive (ER+) breast cancers, up to $40 \%$ of patients are resistant to the traditional endocrine therapy, results from global transcription analysis revealed that transporter SLC6A14 was significantly inhibited by upregulated miR-23b-3p. Moreover, targeting SLC6A14 resensitized the resistant cells to endocrine therapy and impaired their aggressiveness, which provided the promising biomarker and potential target to combat resistant ER+ cells. ${ }^{9}$ A recently published study by Sikder et al proposed that the upregulated SLC6A14 expression in colon cancer was a target of $\mathrm{Wnt} / \beta$-catenin signaling. ${ }^{31}$ To investigate the functional role of SLC6A14 in the pathogenesis of CRC, we also used $\alpha$-MT (a nontransportable blocker) to achieve pharmacologic blockade of SLC6A14 function in AOM-DSS induced CRC models in vivo. We found that blockade of SLC6A14 could protect mice against CRC tumorigenesis by inhibiting cell growth and promoting apoptosis in vivo. Moreover, our in vitro cellular experiments further confirmed the critical role of SLC6A14 in expediting cell proliferation, suppressing apoptosis and promoting metastasis in CRC.

Due to its crucial role in promoting tumor cell proliferation, cell cycle, survival, angiogenesis, epithelialmesenchymal transition (EMT) and immunosuppression, STAT3 has been demonstrated to be a well-characterized driver in many solid tumors including CRC. ${ }^{32,33}$ JAK2 is a well-known upstream kinase to induce STAT3 activity, and JAK2/STAT3 pathway is necessary for the development of colitis-associated tumorigenesis mouse models in vivo. ${ }^{34}$ The newly identified regulators for the activation and functions of JAK2/STAT3 axis are promising therapeutic targets for the treatment of various malignant tumors. Moreover, a remarkable feature of STAT3 as a hopeful target for tumor therapy is that it plays an important role in modulating malignant and immune cells to join hands for immune evasion and treatment resistance. ${ }^{35}$ Besides, STAT3 also acts as a powerful immune checkpoint for many anti-tumor immunity responses. ${ }^{36}$ Although the critical function that STAT3 played in tumor cells and its immune microenvironment is evident, there are still mysteries that remain to be explored in our understanding of the upstream modulators of JAK2/STAT3 signaling. Here, we verified the increasement of JAK2 and STAT3 expression in CRC tumor tissues was positively correlated with the upregulated SLC6A14. And inhibiting JAK2/STAT3 pathway robustly abolished the exacerbation of cell proliferation, colony formation, migration and invasion mediated by exogenous SLC6A14 in CRC cells. Therefore, our data first identified SLC6A14 act as a crucial upstream regulator in modulating the activation of JAK2/STAT3 signaling in the progression of CRC, and our discoveries will pave the new paths to the targeting therapy for patients with CRC.

\section{Conclusions}

In the present study, we found that the aberrantly expressed SLC6A14 in CRC tumor tissues was tightly correlated with lymph node metastasis, advanced TNM stage and poor survival of CRC patients. Functionally, SLC6A14 expedited CRC cell proliferation, suppressed cell apoptosis and promoted mobility in vitro through JAK2/STAT3 signaling. Blocking JAK2/STAT3 signaling specific inhibitors BP-1-102 and FLLL32 markedly ameliorated SLC6A14-induced cancerous phenotypes. Our in vivo experiments also demonstrated that pharmacologic blockade of SLC6A14 function with $\alpha$-MT significantly inhibited intestinal colitis-associated tumorigenesis by suppressing cell growth and inducing cell apoptosis. Besides, SLC6A14 obviously promoted CRC cell growth in vivo, as evidenced by subcutaneous models. Overall, our study highlighted the critical role of SLC6A14 during CRC progression, which may help us to develop a promising therapeutic strategy to block SLC6A14/ JAK2/STAT3 pathway in CRC.

\section{Funding}

This project was supported by the Medical Science and Technology Joint Construction Project of Henan province of China (Grant No. 201901102) and the Science and Technology Project of Henan province of China (Grant No. 192102310133). 


\section{Disclosure}

The authors declare that they have no conflicts of interest.

\section{References}

1. Dekker E, Tanis PJ, Vleugels JLA, Kasi PM, Wallace MB. Colorectal cancer. Lancet. 2019;394(10207):1467-1480. doi:10.1016/S01406736(19)32319-0

2. Keum N, Giovannucci E. Global burden of colorectal cancer: emerging trends, risk factors and prevention strategies. Nat Rev Gastroenterol Hepatol. 2019;16(12):713-732.

3. Marmol I, Sanchez-de-Diego C, Pradilla Dieste A, Cerrada E, Rodriguez Yoldi MJ. Colorectal carcinoma: a general overview and future perspectives in colorectal cancer. Int J Mol Sci. 2017;18 (1):197. doi:10.3390/ijms18010197

4. Bhutia YD, Babu E, Ramachandran S, Ganapathy V. Amino acid transporters in cancer and their relevance to "glutamine addiction": novel targets for the design of a new class of anticancer drugs. Cancer Res. 2015;75(9):1782-1788. doi:10.1158/0008-5472.CAN-14-3745

5. Christensen HN. Role of amino acid transport and countertransport in nutrition and metabolism. Physiol Rev. 1990;70(1):43-77. doi:10.1152/physrev.1990.70.1.43

6. Cormerais Y, Massard PA, Vucetic M, et al. The glutamine transporter ASCT2 (SLC1A5) promotes tumor growth independently of the amino acid transporter LAT1 (SLC7A5). J Biol Chem. 2018;293 (8):2877-2887. doi:10.1074/jbc.RA117.001342

7. El Ansari R, Craze ML, Miligy I, et al. The amino acid transporter SLC7A5 confers a poor prognosis in the highly proliferative breast cancer subtypes and is a key therapeutic target in luminal B tumours. Breast Cancer Res. 2018;20(1):21. doi:10.1186/s13058-018-0946-6

8. Koppula P, Zhang Y, Shi J, Li W, Gan B. The glutamate/cystine antiporter SLC7A11/xCT enhances cancer cell dependency on glucose by exporting glutamate. $J$ Biol Chem. 2017;292 (34):14240-14249. doi:10.1074/jbc.M117.798405

9. Karunakaran S, Ramachandran S, Coothankandaswamy V, et al. SLC6A14 (ATB0,+) protein, a highly concentrative and broad specific amino acid transporter, is a novel and effective drug target for treatment of estrogen receptor-positive breast cancer. $\mathrm{J}$ Biol Chem. 2011;286(36):31830-31838. doi:10.1074/jbc.M111.229518

10. Coothankandaswamy V, Cao S, Xu Y, et al. Amino acid transporter SLC6A14 is a novel and effective drug target for pancreatic cancer. Br J Pharmacol. 2016;173(23):3292-3306. doi:10.1111/bph.13616

11. Penheiter AR, Erdogan S, Murphy SJ, et al. Transcriptomic and immunohistochemical profiling of SLC6A14 in pancreatic ductal adenocarcinoma. Biomed Res Int. 2015;2015:593572. doi:10.1155/ $2015 / 593572$

12. Gupta N, Prasad PD, Ghamande S, et al. Up-regulation of the amino acid transporter ATB $(0,+)$ (SLC6A14) in carcinoma of the cervix. Gynecol Oncol. 2006;100(1):8-13. doi:10.1016/j.ygyno.2005.08.016

13. Gupta N, Miyauchi S, Martindale RG, et al. Upregulation of the amino acid transporter ATB0,+ (SLC6A14) in colorectal cancer and metastasis in humans. Biochim Biophys Acta. 2005;1741(1-2):215-223. doi:10.1016/j.bbadis.2005.04.002

14. Babu E, Bhutia YD, Ramachandran S, et al. Deletion of the amino acid transporter Slc6a14 suppresses tumour growth in spontaneous mouse models of breast cancer. Biochem J. 2015;469(1):17-23. doi:10.1042/BJ20150437

15. Du W, Hong J, Wang YC, et al. Inhibition of JAK2/STAT3 signalling induces colorectal cancer cell apoptosis via mitochondrial pathway. $J$ Cell Mol Med. 2012;16(8):1878-1888. doi:10.1111/j.15824934.2011.01483.x

16. Wei C, Yang C, Wang S, et al. Crosstalk between cancer cells and tumor associated macrophages is required for mesenchymal circulating tumor cell-mediated colorectal cancer metastasis. Mol Cancer. 2019;18(1):64. doi:10.1186/s12943-019-0976-4
17. Johnson DE, O'Keefe RA, Grandis JR. Targeting the IL-6/JAK/ STAT3 signalling axis in cancer. Nat Rev Clin Oncol. 2018;15 (4):234-248.

18. Chu J, Li H, Xing Y, et al. LncRNA MNX1-AS1 promotes progression of esophageal squamous cell carcinoma by regulating miR-34a/ SIRT1 axis. Biomed Pharmacother. 2019;116:109029. doi:10.1016/j. biopha.2019.109029

19. Sun K, Zhao X, Wan J, et al. The diagnostic value of long non-coding RNA MIR31HG and its role in esophageal squamous cell carcinoma. Life Sci. 2018;202:124-130. doi:10.1016/j.lfs.2018.03.050

20. Chu J, Jia J, Yang L, et al. LncRNA MIR31HG functions as a ceRNA to regulate c-Met function by sponging miR-34a in esophageal squamous cell carcinoma. Biomed Pharmacother. 2020;128:110313. doi:10.1016/j.biopha.2020.110313

21. Yang L, Ye Y, Chu J, et al. Long noncoding RNA FEZF1-AS1 promotes the motility of esophageal squamous cell carcinoma through Wnt/beta-catenin pathway. Cancer Manag Res. 2019;11:4425-4435. doi:10.2147/CMAR.S196004

22. Fuchs BC, Bode BP. Amino acid transporters ASCT2 and LAT1 in cancer: partners in crime? Semin Cancer Biol. 2005;15(4):254-266. doi:10.1016/j.semcancer.2005.04.005

23. He J, Zhou M, Li X, et al. SLC34A2 simultaneously promotes papillary thyroid carcinoma growth and invasion through distinct mechanisms. Oncogene. 2020;39(13):2658-2675. doi:10.1038/ s41388-020-1181-Z

24. He J, Jin Y, Zhou M, et al. Solute carrier family 35 member F2 is indispensable for papillary thyroid carcinoma progression through activation of transforming growth factor-beta type I receptor/apoptosis signal-regulating kinase 1 /mitogen-activated protein kinase signaling axis. Cancer Sci. 2018;109(3):642-655. doi:10.1111/cas.13478

25. Morioka S, Perry JSA, Raymond MH, et al. Efferocytosis induces a novel SLC program to promote glucose uptake and lactate release. Nature. 2018;563(7733):714-718. doi:10.1038/s41586-018-0735-5

26. Zhang Y, Zhang Y, Sun K, Meng Z, Chen L, Liu F. The SLC transporter in nutrient and metabolic sensing, regulation, and drug development. J Mol Cell Biol. 2019;11(1):1-13. doi:10.1093/jmcb/mjy052

27. White MA, Frigo DE. Regulation of SLC1A4 and SLC1A5 in prostate cancer-response. Mol Cancer Res. 2018;16(11):1811-1812. doi:10.1158/1541-7786.MCR-18-0240

28. van Geldermalsen M, Wang Q, Nagarajah R, et al. ASCT2/SLC1A5 controls glutamine uptake and tumour growth in triple-negative basal-like breast cancer. Oncogene. 2016;35(24):3201-3208. doi:10.1038/onc.2015.381

29. Salisbury TB, Arthur S. The regulation and function of the L-type amino acid transporter 1 (LAT1) in cancer. Int J Mol Sci. 2018;19 (8):2373. doi:10.3390/ijms19082373

30. Lang X, Green MD, Wang W, et al. Radiotherapy and immunotherapy promote tumoral lipid oxidation and ferroptosis via synergistic repression of SLC7A11. Cancer Discov. 2019;9(12):1673-1685. doi:10.1158/2159-8290.CD-19-0338

31. Sikder MOF, Sivaprakasam S, Brown TP, Thangaraju M, Bhutia YD, Ganapathy V. SLC6A14, a Na+/Cl-coupled amino acid transporter, functions as a tumor promoter in colon and is a target for Wnt signaling. Biochem J. 2020;477(8):1409-1425. doi:10.1042/BCJ20200099

32. Yang L, Zhang R, Yang J, Bi T, Zhou S. FKBP14 promotes the proliferation and migration of colon carcinoma cells through targeting IL-6/STAT3 signaling pathway. Onco Targets Ther. 2019;12:9069-9076. doi:10.2147/OTT.S222555

33. Li P, Huang Z, Wang J, Chen W, Huang J. Ubiquitin-specific peptidase 28 enhances STAT3 signaling and promotes cell growth in non-small-cell lung cancer. Onco Targets Ther. 2019;12:1603-1611. doi:10.2147/OTT.S194917

34. Bollrath J, Phesse TJ, von Burstin VA, et al. gp130-mediated Stat3 activation in enterocytes regulates cell survival and cell-cycle progression during colitis-associated tumorigenesis. Cancer Cell. 2009;15(2):91-102. doi:10.1016/j.ccr.2009.01.002 
35. Wang Z, Yin M, Wang R, Liu X, Yan D. Bit1 silencing enhances the proliferation, migration, and invasion of glioma cells through activation of the IL-6/STAT3 pathway. Onco Targets Ther. 2020;13:2469-2481. doi:10.2147/OTT.S240081
36. Kortylewski M, Kujawski M, Wang T, et al. Inhibiting Stat3 signaling in the hematopoietic system elicits multicomponent antitumor immunity. Nat Med. 2005;11(12):1314-1321. doi:10.1038/nm1325

\section{Publish your work in this journal}

OncoTargets and Therapy is an international, peer-reviewed, open access journal focusing on the pathological basis of all cancers, potential targets for therapy and treatment protocols employed to improve the management of cancer patients. The journal also focuses on the impact of management programs and new therapeutic

Submit your manuscript here: https://www.dovepress.com/oncotargets-and-therapy-journal agents and protocols on patient perspectives such as quality of life, adherence and satisfaction. The manuscript management system is completely online and includes a very quick and fair peer-review system, which is all easy to use. Visit http://www.dovepress.com/ testimonials.php to read real quotes from published authors. 